\title{
Modelling All-Optical Switching and Limiting Properties of AlAs Photonic Crystals
}

\author{
M.G. Pravini S. Fernando ${ }^{1, a}$, K.A.I.L.Wijewardena Gamalath ${ }^{2, b^{*}}$ \\ ${ }^{1,2}$ Department of Physics University of Colombo, Sri Lanka \\ apravifdo@gmail.com, ${ }^{*}$ imalie@phys.cmb.ac.lk
}

\begin{abstract}
Keywords: Photonic crystals, All-Optical switch, Limiter, FDTD, Kerr nonlinearity, AIAs and Side coupled Micro cavities
\end{abstract}

\begin{abstract}
The incorporation of defect modes into the perfect crystal structure allows the control of the flow of light by altering the photonic bandgap and thereby can be manipulated to achieve optical switching. A model for all optical switching and limiting based on two dimensional photonic crystals is proposed for AlAs and the performance in square and hexagonal lattice structures were evaluated. Simulations were done using 2D finite difference time domain model incorporating instantaneous Kerr's nonlinearity. The optimal nonlinear resonant frequencies and the refractive index change required for the performance in the nonlinear regime were obtained. The limiter effectiveness is analysed using extinction ratio. The lattice constant and the optimal microcavity distance required for the proposed model to work as a switch and a limiter in the telecommunication wavelength of $1.55 \mu \mathrm{m}$ were obtained as $\mathrm{a}=0.6015 \mu \mathrm{m}$ and $2 \mathrm{a}$ respectively.
\end{abstract}

\section{Introduction}

Photonic crystals (PCs) are materials with index of refraction varying periodically between high-index regions and low-index regions giving rise to a range of forbidden frequencies called photonic bandgaps analogues to electronic bandgaps in semiconductors [1]. There are three types of PCs depending on one, two or three-dimensional arrangement in periodicity [2]. Although periodic arrangement of atoms within a semiconductor material occurs in nature such as opal gemstones, beetles to bird feathers and butterfly wings, PCs are mostly artificially fabricated. For frequency energies lying within the bandgap, the photonic crystals do not allow light to propagate through unless there is a defect in the otherwise perfect crystal. The introduction of various types of defects, provide the ability to guide and mould the flow of light or photons propagation through the gap. Linear defects can act as efficient waveguides [3] while point like defects may act as resonant micro cavities coupling light to photonic band gap (PBG) waveguides and other optical components [4]. Several studies had been carried out using external modifications of material's refractive index to change the band-gap structure such as by temperature variations [5] and electro-optic method [6]. However, these are slow in comparison with the speed required for modern communication.

The field of nonlinear optics, the propagation of light in nonlinear media having nonlinear relation between dielectric polarization $(\boldsymbol{P})$ and electric field $(\boldsymbol{E})$ emerged with the discovery of second-harmonic generation by Franken et al. in 1961 [7]. When nonlinearity is incorporated into a photonic crystal, the light propagation can be controlled dynamically [8]. This field has grown enormously contributing vastly to the advancement of the telecommunication industry and opened up opportunities to design all-optical devices [9]. Kerr nonlinearity is an instantaneous nonlinear response that results a dependence of refractive index on the light intensity. This occurs when intense light propagates in media such as crystals, glass and gases and holds a great importance due to its suitability in ultra-fast devices. Several structures of PC switches have been implemented over the years incorporating Kerr's nonlinearity [10, 11, 12].

The principle of optical switching and limiting was demonstrated in a one-dimension (1D) by Scalora and Tran in 1993 [10] by modelling both the probe and the pump pulses propagating in the same direction. Using Kerr nonlinearity Scholz et al. designed a two-dimensional optical switch which incorporated the crossed wave guides [11] and due to the perpendicular directions of 
propagation of the probe beam and the pump pulse, the undesirable overlay of the two signals was reduced. Experiments have been carried out demonstrating all-optical switching action in nonlinear PC cross-waveguide geometry of AlGaAs with Kerr nonlinearity and the transmission of a signal that can be reversibly switch on and off by a control input [13] and thereby accomplishing both spatial and spectral separation between the signal and the control in nonlinear regime. An all optical switch based on nonlinear PC microcavities for AlGaAs was demonstrated numerically based on Finite Difference Time Domain (FDTD) incorporating Kerr effect [14]. Many suggestions have been brought up in implementing optical switches using directional couplers $[12,15,16]$ by modifying 2D PC structures by creating two single line defect waveguides adjacent to each other, separated by the single row of rods with decreased radius [12]. In 2008, numerical experiments were carried out investigating the possibility of the radiation intensity stabilization using the 2D PC structures [17] approving the possibility of designing optical power limiter circuits using 2D PCs. Danaie and his colleagues designed a PC optical limiter using nonlinear Kerr Effect for a triangular lattice of holes in a GaAs substrate [18]. Optical limiters have been incorporated in designing nonlinear optical devices such as AND gates using Kerr nonlinearity [19].

To model nonlinearity, finite difference time domain method (FDTD), finite element method (FEM), plane wave expansion (PWE) and Wannier function method (WFM) can be used in incorporating nonlinear elements into the linear system. FDTD method, which enables modelling time evolution of the fields and quantities in the desired structure [20] is regarded as one of the most popular numerical methods used to simulate nonlinearity and dispersion structure. FDTD further enables to model different geometries in different dimensions. FDTD was first proposed by Yee in 1966 [21]. This method proposed a discrete solution to Maxwell's Equations based on central difference approximations of the spatial and temporal derivatives of the curl operations. It has been developed to a fast algorithm to solve sophisticated problems. An underlying weakness of this method is that it requires a full discretization of the electric and the magnetic fields in the entire volume domain [22].

This work investigates nonlinear optical properties of PCs in optical switching and limiting. 2D PCs with $15 \times 15$ square lattice of AlAs rods with air wafer dimensions $17 \mu \mathrm{m} \times 16 \mu \mathrm{m}$ and lattice constant of $\mathrm{a}=1 \mu \mathrm{m}$ and $15 \times 15$ hexagonal lattice structures of AlAs rods with the air wafer dimensions $17 \mu \mathrm{m} \times 14 \mu \mathrm{m}$ and lattice constants $\mathrm{a}=0.866 \mu \mathrm{m}$ and $\mathrm{b}=1 \mu \mathrm{m}$ were considered without loss of generality. Kerr nonlinearity was incorporated and a simulation was carried out based on FDTD method. An optical switch and an optical limiter are proposed using a side-coupled cavity waveguide for AlAs along with fine tuning to work under the telecommunication wavelength.

\section{Effects of Nonlinearity}

Optical nonlinearity is a phenomenon which occurs due to modification of optical properties of a material by the presence of light. Ideally, only laser light in this regard has a sufficient intensity to modify optical properties of material [23]. The propagation of light in a photonic crystal is governed by the macroscopic Maxwell equations:

$$
\nabla \times \boldsymbol{E}=-\frac{\partial \boldsymbol{B}}{\partial t} ; \quad \nabla \times \boldsymbol{H}=\boldsymbol{J}+\frac{\partial \boldsymbol{D}}{\partial t} ; \quad \nabla \cdot \boldsymbol{B}=0 ; \quad \nabla \cdot \boldsymbol{E}=\rho,
$$

where $\rho$ and $\boldsymbol{J}$ are free electron charge density and current density respectively. The relationships between the four electromagnetic field vectors $\boldsymbol{E}, \boldsymbol{D}, \boldsymbol{B}, \boldsymbol{H}$ are given by:

$$
\boldsymbol{D}=\varepsilon_{0} \boldsymbol{E}+\boldsymbol{P} ; \quad \boldsymbol{B}=\mu_{0}(\boldsymbol{H}+\boldsymbol{M}),
$$

where $\boldsymbol{P}$ is the polarization field and $\boldsymbol{M}$ is the magnetization field. Inside a dielectric, the induced polarization $P$ of the medium is given by [23]:

$$
P=\varepsilon_{o} \chi^{(1)} E+\varepsilon_{o} \chi^{(2)} E^{2}+\varepsilon_{o} \chi^{(3)} E^{3}+\mathrm{L}
$$


Here $\chi^{(1)}, \chi^{(2)}$ and $\chi^{(3)}$ are called the first, second and third order susceptibility coefficients respectively. $\chi^{(2)}$ is only observed in non-centrosymmetric crystals, hence liquids, gas and most of the solids do not possess this susceptibility. On the other hand, third-order susceptibility occurs for both centrosymmetric and non-centrosymmetric media and most of the materials display this susceptibility which gives rise to Kerr nonlinearity. A monochromatic electric field $E=E_{\omega} \cos \omega t$ induced polarization of the medium [24]:

$$
P \approx \varepsilon_{0}\left(\chi^{(1)}+\frac{3}{4} \chi^{(3)}\left|E_{\omega}\right|^{2}\right) E_{\omega} \cos \omega t
$$

Here the formula $\cos ^{3} \omega t=(\cos 3 \omega t+3 \cos \omega t) / 4$ is used. Resultant susceptibility can be considered to be a summation of linear $\chi_{L}$ and nonlinear $\chi_{N L}$ components. The index of refraction:

$$
n=(1+\chi)^{\frac{1}{2}}=\left(1+\chi_{L}+\chi_{N L}\right)^{\frac{1}{2}} \approx n_{0}\left(1+\frac{\chi_{N L}}{2 n_{0}^{2}}\right)=n_{0}+\left(\frac{3 \chi^{(3)}}{8 n_{0}}\right) \times\left|E_{\omega}\right|^{2}=n_{0}+n_{2} I
$$

where $n_{0}=\sqrt{1+\chi_{L}}$ is the linear refractive index, $n_{2}$ is the second order refractive index and $I$ is the intensity of light. The electric field and displacement fields are now related by:

$$
D(t)=\varepsilon_{0} \varepsilon_{r} E(t)=\varepsilon_{0} n^{2} E(t)=\varepsilon_{0}\left(n_{0}{ }^{2}+\frac{3}{4} \chi^{(3)}|E(t)|^{2}\right) E(t)
$$

The most commonly used time-domain differential equation approach in computational electrodynamics (CEM), is finite difference time domain method (FDTD) and it mainly helps in solving time dependent Maxwell's equations given in equation 1 with:

$$
\boldsymbol{D}=\varepsilon_{0} \varepsilon_{r} \boldsymbol{E} ; \quad \boldsymbol{B}=\mu_{0} \mu_{r} \boldsymbol{H}
$$

Electric fields are normalized so that the $\boldsymbol{E}$-field and the $\boldsymbol{H}$-field have the same order of magnitude. This has an advantage in formulating the perfectly matched layer (PML) which is a crucial part of FDTD simulation. Hence, the normalized electric fields are introduced as:

$$
\tilde{\boldsymbol{E}}=\sqrt{\frac{\mu_{0}}{\mu_{0}}} \boldsymbol{E}, \quad \tilde{\boldsymbol{D}}=\frac{\boldsymbol{D}}{\sqrt{\mu_{0} \varepsilon_{0}}}=c \boldsymbol{D}
$$

The Maxwell's equations with normalized $\boldsymbol{E}$-fields read:

$$
\nabla \times \tilde{\boldsymbol{E}}=-\frac{\mu_{r}}{c} \frac{\partial \boldsymbol{B}}{\partial t} ; \quad \nabla \times \boldsymbol{H}=\frac{1}{c} \frac{\partial \tilde{\boldsymbol{D}}}{\partial t}
$$

These equations are independent of the material in use and hence lead to lesser complications in deriving the equations in the main FDTD algorithm. The constitutional relationship which related the $\boldsymbol{E}$ and the $\boldsymbol{D}$-fields will account for the material properties which are given by the Kerr's nonlinearity. Assuming that the relative permeability of the material $\left(\mu_{r}\right)$ is diagonal and the $z$ direction is uniform and of infinite extent (i.e. $\partial / \partial z=0$ ), equations in 9 can be expanded as follows: 


$$
\begin{array}{ll}
\frac{\partial H_{z}}{\partial y}=\frac{1}{c} \frac{\partial \tilde{D}_{x}}{\partial t}=C_{x}^{H} & \frac{\partial \tilde{E}_{z}}{\partial y}=-\frac{\mu_{x x}}{c} \frac{\partial H_{x}}{\partial t}=C_{x}^{E} \\
-\frac{\partial H_{z}}{\partial x}=\frac{1}{c} \frac{\partial \tilde{D}_{y}}{\partial t}=C_{y}^{H} & -\frac{\partial \tilde{E}_{z}}{\partial x}=-\frac{\mu_{y y}}{c} \frac{\partial H_{y}}{\partial t}=C_{y}^{E} \\
\frac{\partial H_{y}}{\partial x}-\frac{\partial H_{x}}{\partial y}=\frac{1}{c} \frac{\partial \tilde{D}_{z}}{\partial t}=C_{z}^{H} & \frac{\partial \tilde{E}_{z}}{\partial x}-\frac{\partial \tilde{E}_{x}}{\partial y}=-\frac{\mu_{z z}}{c} \frac{\partial H_{z}}{\partial t}=C_{z}^{E}
\end{array}
$$

These two sets of equations corresponds to the TM mode ( $E_{z}$ mode) and the TE mode $\left(H_{z}\right)$ mode respectively. In this paper we consider photonic crystal behaviour in TM mode only and therefore the curl elements of the TM mode equations in 2-D finite differences are rearranged in order to obtain the basic TM mode field update equations of the main FDTD algorithm:

$$
\begin{aligned}
& \left.H_{x}\right|_{t+\Delta t / 2} ^{i, j}=\left.H_{x}\right|_{t-\Delta t / 2} ^{i, j}-\left.\frac{c \Delta t}{\mu_{x x}} C_{x}^{E}\right|_{t} ^{i, j} ;\left.\quad H_{y}\right|_{t+\Delta t / 2} ^{i, j}=\left.H_{y}\right|_{t-\Delta t / 2} ^{i, j}-\left.\frac{c \Delta t}{\mu_{y y}} C_{y}^{E}\right|_{t} ^{i, j} \\
& \left.\tilde{D}_{z}\right|_{t+\Delta t} ^{i, j}=\left.\tilde{D}_{z}\right|_{t} ^{i, j}+\left.c \Delta t C_{z}^{H}\right|_{t+\Delta t} ^{i, j}
\end{aligned}
$$

In order to avoid reflections, perfectly matched layer (PML) boundaries are incorporated into FDTD algorithm to avoid reflections at the boundaries. In the simulation, the imperfect truncation of the problem space will create numerical reflections which will corrupt the computational results in the problem space after some time. The perfectly matched layer (PML) introduced by Berenger in 1994 [25] has been proven to be one of the most strong absorbing boundary condition (ABCs) when compared to other techniques used in the past [26], [27]. PML is a finite-thickness layer of special medium surrounding the computational space based on fictitious constitutive parameters to create a wave-impedance matching condition, which is independent of the angles and frequencies of the wave incident on this boundary. The PML boundaries were incorporated to the main FDTD algorithm by the method proposed by Zachary et al. [28]. Time increment $(\Delta t)$ was obtained by separating out the finite-difference algorithm into separate time and space eigenvalue problems by enforcing the stability condition called the Courant-Friedrich-Levy (CFL) or Courant's stability condition [29]:

$$
\Delta t=\frac{n_{\min } \min [\Delta x, \Delta y]}{2 c}
$$

\section{Switch Coupler Structure Geometry Analysis}

Two dimensional photonic crystals were designed for the square and hexagonal geometries. Hexagonal structure was composed of $15 \times 15$ hexagonal lattice of AlAs rods with the air wafer dimensions $17 \mu \mathrm{m} \times 14 \mu \mathrm{m}$ and lattice constants $\mathrm{a}=0.866 \mu \mathrm{m}$ and $\mathrm{b}=1 \mu \mathrm{m}$ while the square lattice structure was composed of $15 \times 15$ square lattice of AlAs rods with air wafer dimensions $17 \mu \mathrm{m} \times 16 \mu \mathrm{m}$ and lattice constant of $\mathrm{a}=1 \mu \mathrm{m}$. The AlAs rods has the linear refractive index $\left(n_{L}\right)$ 2.892 at wavelength $1550 \mathrm{~nm}$ [30]. For band gap calculations Optiwave PWE (plane wave expansion) band solver software was used. A line defect to the crystal structure was introduced by removing a single row of AlAs rods. The waveguides created for both structures are shown in Fig. 1. The transmittance of radiation for the power variation of $0-1.6 \times 10^{-13} \mathrm{Wm}^{-1}$ through the wave guide were measured for the change in the radii of the AlAs rods from $0.1 \mathrm{a}$ to $0.4 \mathrm{a}$. The normalized transmission spectra for different radii for the square and hexagonal lattice structures with a single waveguide are presented in Fig. 2. The radii range which gave the maximum transmission corresponds to $0.13 \mathrm{a}-0.25 \mathrm{a}$ and $0.14 \mathrm{a}-0.28 \mathrm{a}$ for square and hexagonal lattice structures respectively. The radius $0.1875 \mathrm{a}$ was found to be the best value which gave the 
maximum output power for the square lattice structure while $0.2050 \mathrm{a}$ was considered as the best radii for the hexagonal structure. Hence, circular cylindrical pillars of the photonic crystal structures were changed to the best radius value and the transverse magnetic (TM) band gap of this structure was obtained by the PWE method.
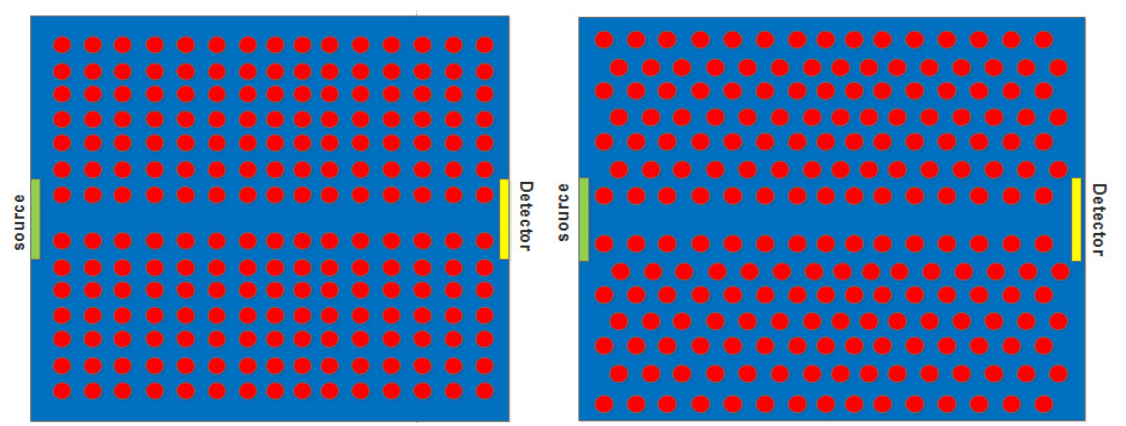

Figure 1. Photonic crystal with line defects. (Left) Square lattice (Right) Hexagonal lattice.

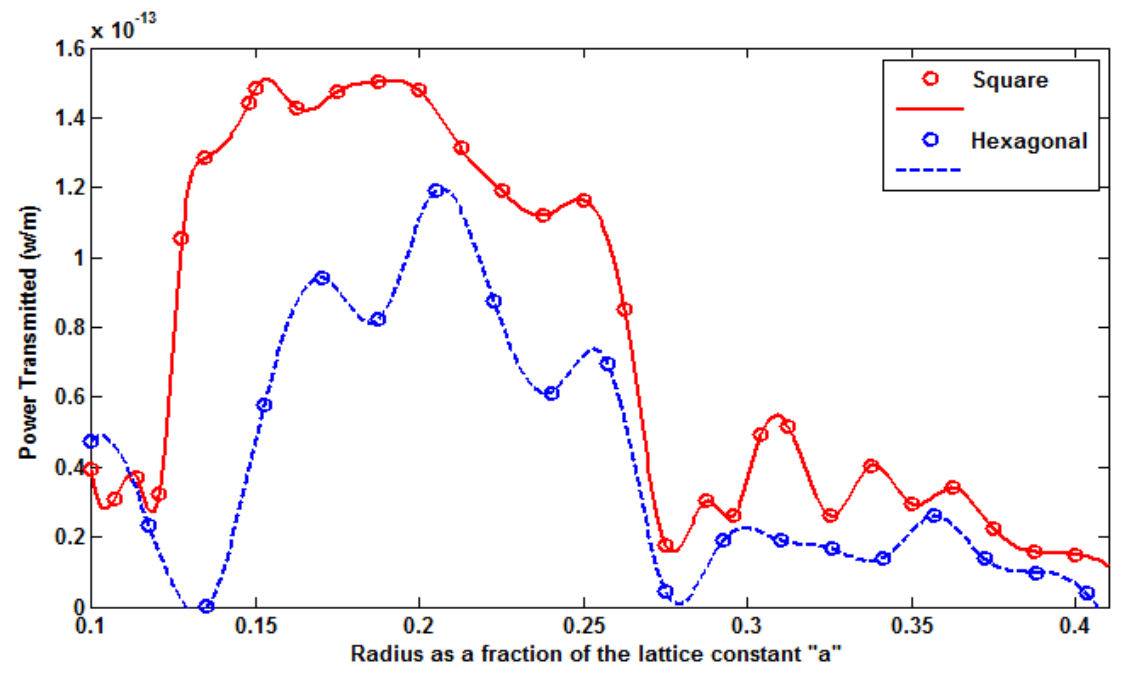

Figure 2. The power transmitted for different radii of square and hexagonal PC lattice waveguide structures of AlAs.

\section{Square PC Lattice Structure}

For the given parameters of the square PC lattice structure, the band gap for the TM mode is $0.112638 / \lambda$ and the gap lies in the range of normalized frequencies $0.345 \leq 1 / \lambda \leq 0.457$ where $\lambda$ denotes the optical wavelength in the free space. Forbidden bands of wavelengths called photonic band gaps give rise to distinct optical phenomena which can be used to obtain controlled photon behaviour. The lattice was modified thrice by introducing a microcavity by removing a single AlAs rod at distances $\mathrm{a}, 2 \mathrm{a}$ and $3 \mathrm{a}$ from the middle of the waveguide separately. The wavelengths corresponding to the respective normalized frequency bandgap $2.188 \mu \mathrm{m}-2.899 \mu \mathrm{m}$ were transmitted through the source point and the transmittance at each scenario was recorded. Fig. 3 shows the normalized transmission spectra for the three cases, a, 2a and $3 \mathrm{a}$. In all the three cases, there are sudden drops of the transmission powers for certain wavelength values. These sudden drops occur when the frequency of the incoming signal resonates with the resonance frequency of the microcavity. At this instance the incoming wave couples with the microcavity and hence the transmission through the waveguide is seized. These resonance wavelengths and the corresponding resonance frequencies of the cavity are tabulated in table 1 . The simulation results for AlAs square PC crystal switch structure with microcavity at distances a, 2a and $3 \mathrm{a}$ from the wave guide at the resonant wavelengths are presented in Figs. 4: $(a),(b)$ and $(c)$ respectively while figs. 4: $(d),(e)$ and $(f)$ represents the simulation results at $2.600 \mu \mathrm{m}$ wavelength for microcavity distances a, $2 \mathrm{a}$ and $3 \mathrm{a}$ respectively. 


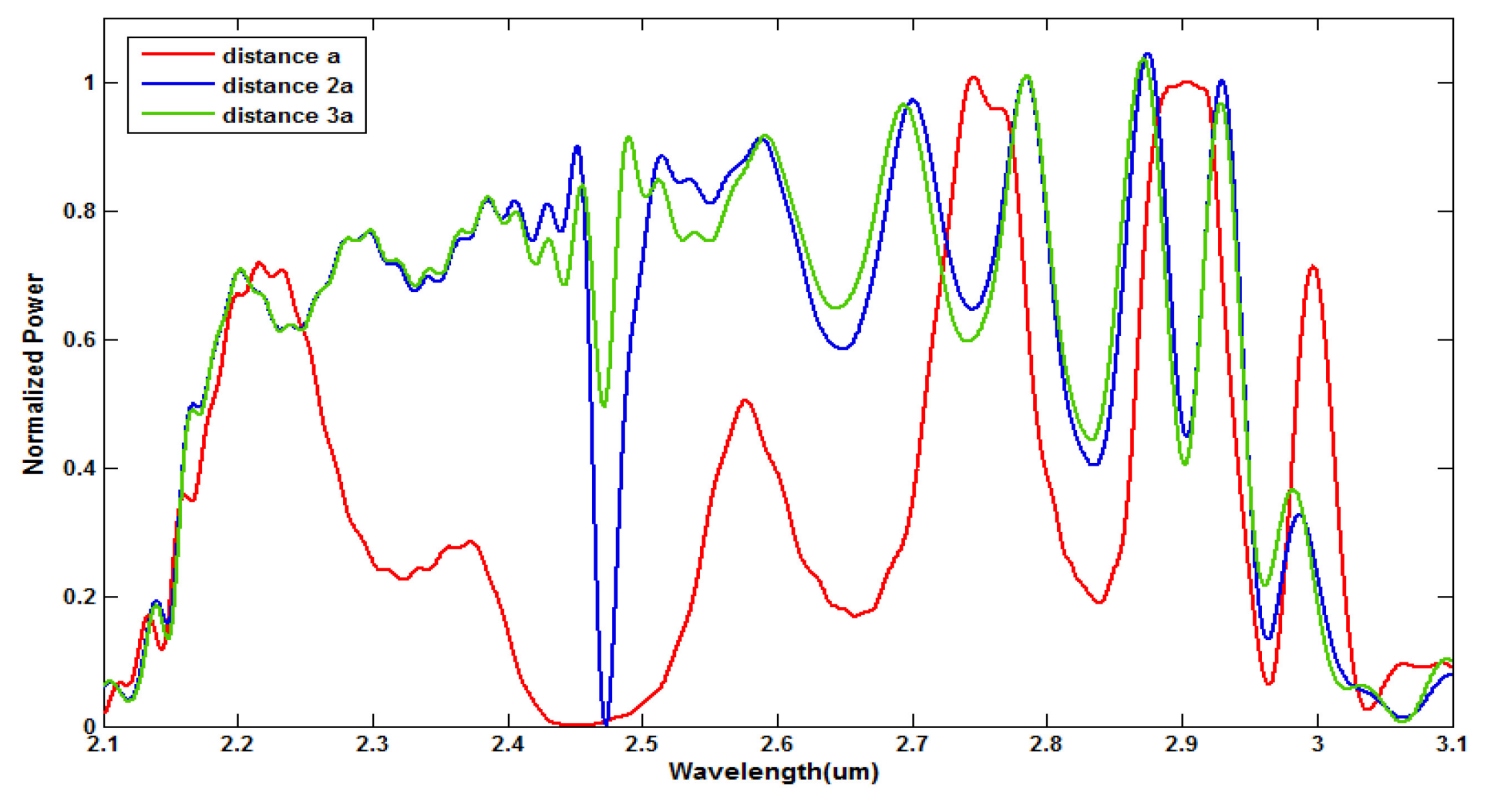

Figure 3. The normalized transmission spectra for the PC square lattice switch structure for 3 different microcavity distances a, $2 \mathrm{a}$ and $3 \mathrm{a}$ from the middle of the waveguide.

Table 1. Resonance frequencies for each of the cavity distances a, $2 \mathrm{a}$ and $3 \mathrm{a}$ with the normalized transmission powers detected for AlAs square PC lattice.

\begin{tabular}{|c|c|c|}
\hline Cavity distance $(\mathrm{a})$ & Resonance wavelength $(\mu \mathrm{m})$ & Normalized transmission \\
\hline $\mathrm{a}$ & 2.448 & $1.174 \times 10^{-4}$ \\
\hline $2 \mathrm{a}$ & 2.470 & $1.094 \times 10^{-3}$ \\
\hline $3 \mathrm{a}$ & 2.472 & 0.496 \\
\hline
\end{tabular}

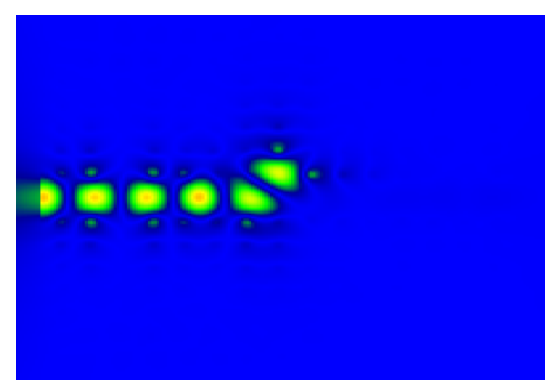

(a)

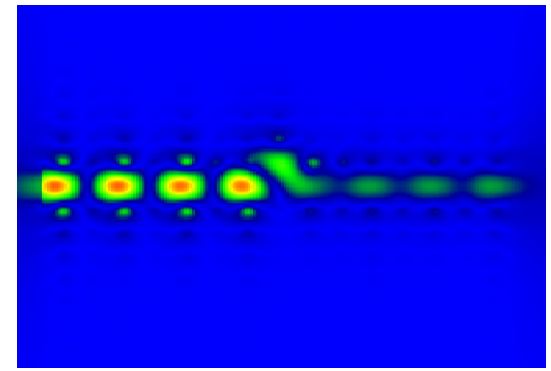

(d)

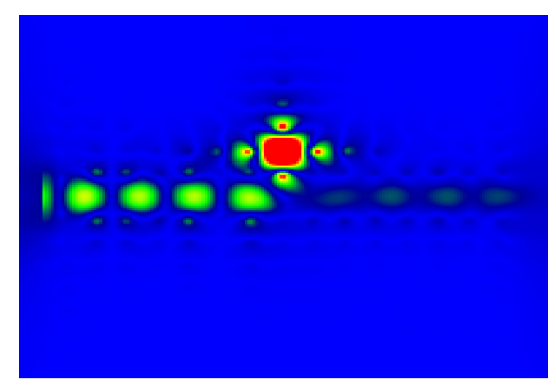

(b)

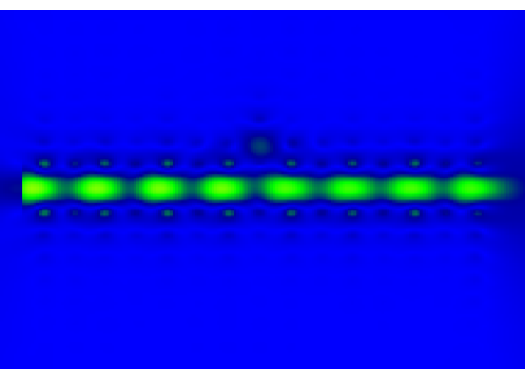

(e)

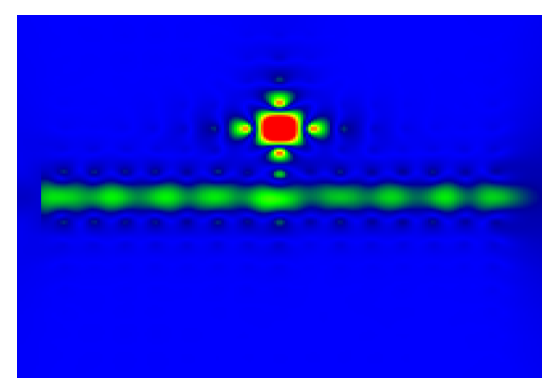

(c)

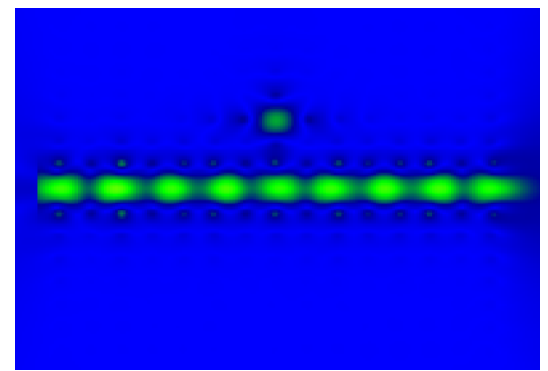

(f)

Figure 4. Simulation results of AlAs square PC crystal switch structure with microcavity at distances a, $2 \mathrm{a}$ and $3 \mathrm{a}$ from the wave guide. $(a),(b)$ and $(c)$ represents the simulation results at the resonant wavelengths and figures $(d),(e)$ and $(f)$ represents the simulation results at $2.600 \mu \mathrm{m}$ wavelength for microcavity distances a, $2 \mathrm{a}$ and $3 \mathrm{a}$ respectively.

From Fig. 4 it is clear that when the microcavity is at a distance 'a' away from the waveguide, the normalized transmittance power along the waveguide at the resonance is minimum value of the order $10^{-4}$ compared to Figs. 4(b) and Fig. 4(c). However, at $2.600 \mu \mathrm{m}$ wavelength fig. 4(d) shows 
a less intense beam being propagated along the waveguide with a normalized transmission power of 0.383 compared to the fig. 4(e) and fig. 4(f). Hence the optical switch formed from AlAs square switching structure with the microcavity at a distance ' $a$ ' from the waveguide shows poor performance. PC structure with the microcavity at a distance $2 \mathrm{a}$ from the waveguide has a sharp drop of transmission power at resonance to an order of $10^{-3}$. Fig. 4(b) shows that at resonance the incoming wave couples well with the microcavity. At other wavelengths the incoming wave goes through the waveguide without being coupled to the resonance cavity. The intense propagation of normalized transmission of 0.839 in fig. 4(e) further proves this. When the microcavity is at a distance $3 \mathrm{a}$ from the waveguide the resonance occurs with a slight drop of transmission. Normalized transmission in this instance was 0.496. Fig. 4(c) shows that there is a comparably intense output together with coupling to the microcavity. Hence, this structure too shows poor performance at resonance. Therefore for the square lattice structure of AlAs, the best optical switching can be obtained for rods of radius $0.1875 \mathrm{a}$ with a resonance wavelength of $2.470 \mu \mathrm{m}$ when the microcavity is at a distance $2 \mathrm{a}$ from the waveguide.

\section{Hexagonal PC Lattice Structure}

For the given parameters of the hexagonal PC lattice structure, the band gap for the TM mode is $0.160954 / \lambda$ and the gap lies in the range of normalized frequencies $0.321 \leq 1 / \lambda \leq 0.481$ where $\lambda$ denotes the optical wavelength in the free space. The lattice was modified by introducing a microcavity by removing a single AlAs rod at distances a, $2 \mathrm{a}$ and $3 \mathrm{a}$ from the middle of the waveguide separately. Thereby, wavelengths corresponding to the respective normalized frequency bandgap $2.08-3.11 \mu \mathrm{m}$ were transmitted through the source point and the transmittance for each case was recorded and Fig. 5 shows the normalized transmission spectra for these three cases. The resonance wavelengths and the normalized transmission detected at resonance are tabulated in Table 2.

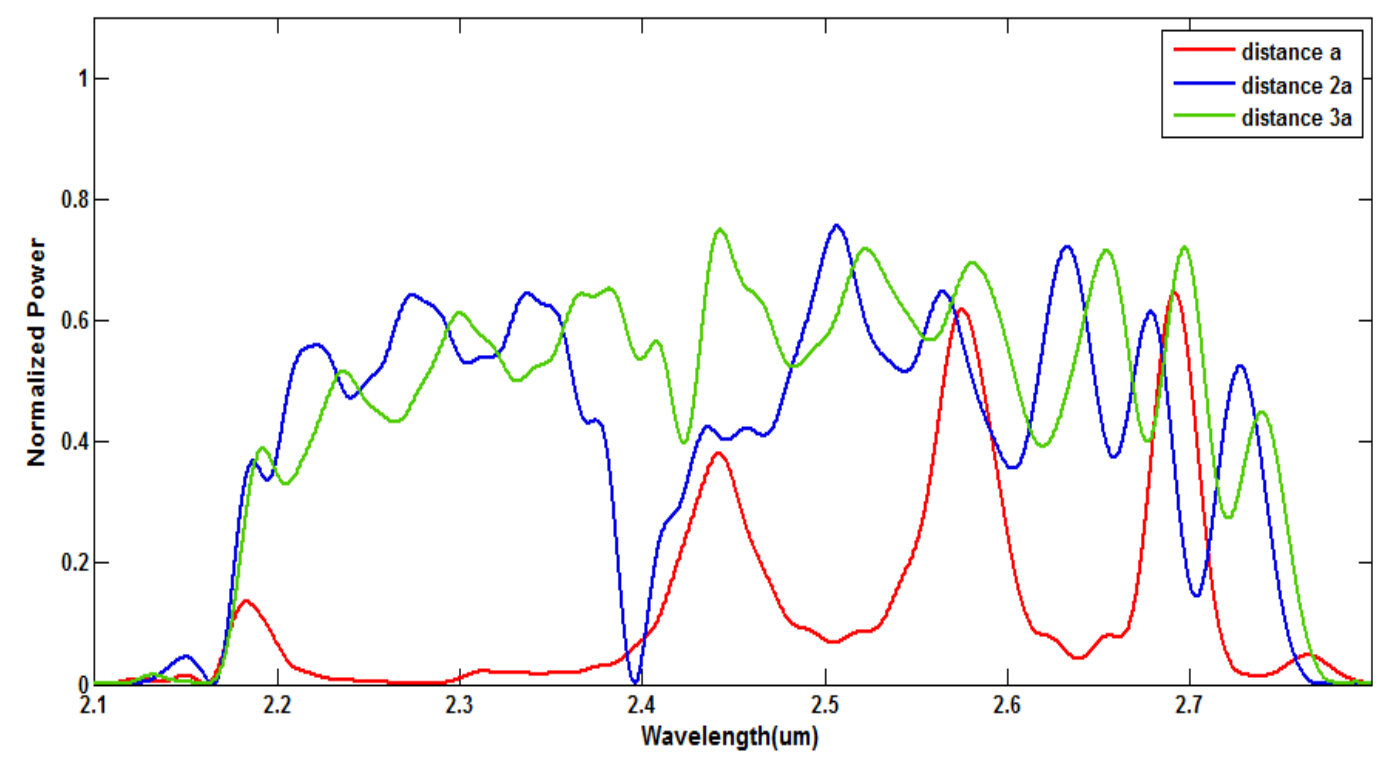

Figure 5. Variation of the normalized transmission spectra for the hexagonal PC lattice switch structure for microcavity distances $\mathrm{a}, 2 \mathrm{a}$ and $3 \mathrm{a}$ from the middle of the waveguide.

Table 2. Resonance frequencies for the cavity distances a, $2 \mathrm{a}$ and $3 \mathrm{a}$ with the corresponding normalized transmission detected for AlAs Hexagonal PC lattice.

\begin{tabular}{|c|c|c|}
\hline Cavity distance (a) & Resonance wavelength $(\mu \mathrm{m})$ & Normalized transmission \\
\hline $\mathrm{a}$ & 2.282 & $3.757 \times 10^{-5}$ \\
\hline $2 \mathrm{a}$ & 2.396 & $2.764 \times 10^{-3}$ \\
\hline $3 \mathrm{a}$ & 2.422 & 0.4011 \\
\hline
\end{tabular}




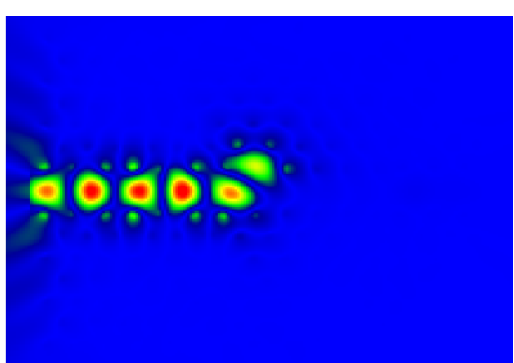

(a)

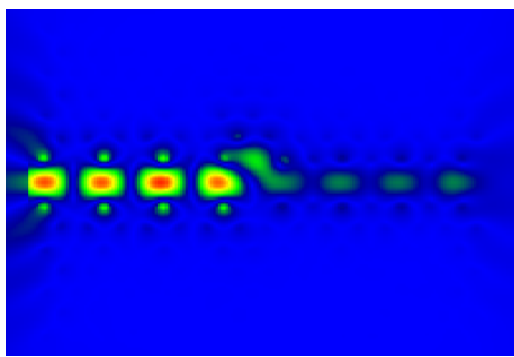

(d)

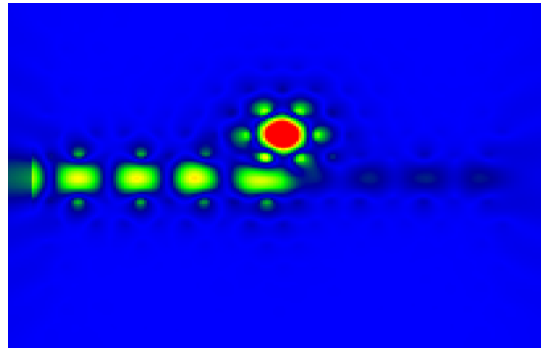

(b)

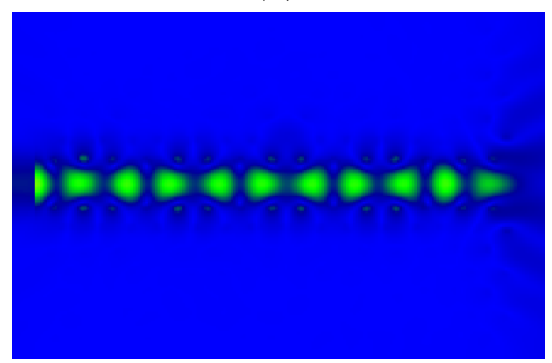

(e)

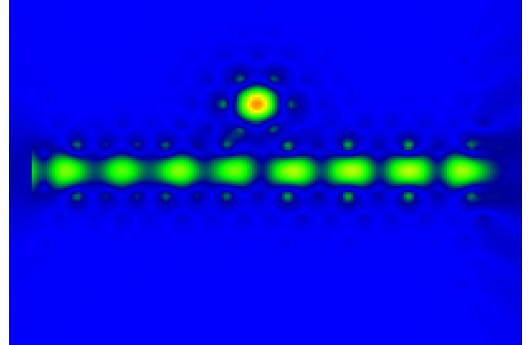

(c)

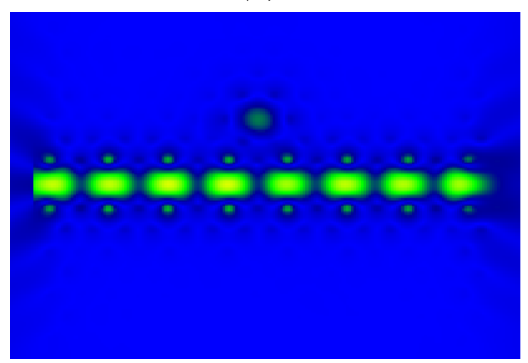

(f)

Figure 6. Simulation results of AlAs Hexagonal PC crystal switch structure with microcavity distances at $\mathrm{a}, 2 \mathrm{a}$ and $3 \mathrm{a}$ distances from the wave guide. $(a),(b)$ and $(c)$ represents the simulation results at the resonant wavelengths and figures $(d),(e)$ and $(f)$ represents the simulation results at $2.600 \mu \mathrm{m}$ wavelength for microcavity distances a, $2 \mathrm{a}$ and $3 \mathrm{a}$.

The simulations for AlAs hexagonal PC crystal switch structure with microcavity at distances $\mathrm{a}, 2 \mathrm{a}$ and $3 \mathrm{a}$ from the wave guide at the resonant wavelengths are presented in figs. $6:(a),(b)$ and (c) respectively while Figs. 6: $(d),(e)$ and $(f)$ represents the simulation results at $2.600 \mu \mathrm{m}$ wavelength for microcavity distances a, $2 \mathrm{a}$ and $3 \mathrm{a}$ respectively. When the microcavity is at ' $\mathrm{a}$ ' distance away from the waveguide, the transmittance at the resonance is a minimum $\left(\sim 10^{-5}\right)$ along the waveguide compared to Fig. $6(b)$ and Fig. 6(c). At wavelength $2.600 \mu \mathrm{m}$ Fig. $6(d)$ shows a less intense beam being propagated along the waveguide compared to Fig. 6(e) and Fig. 6(f). Hence, optical switch formed from AlAs hexagonal structure with the microcavity at a distance 'a' from the waveguide shows poor performance. When the microcavity at a distance $2 \mathrm{a}$ from the waveguide, transmission power has a sharp drop at resonance with transmittance of an order of $10^{-3}$. Fig. $6(b)$ shows that at resonance, the incoming wave couples well with the microcavity. The intense propagation of the beam in Fig. 6(d) at $2.600 \mu \mathrm{m}$ wavelength shows that the incoming wave goes through the waveguide without being coupled to the resonance cavity. When the microcavity is at a distance $3 \mathrm{a}$ from the waveguide the resonance occurs with a slight drop of transmission. Normalized transmission in this instance was 0.4011. Fig. 6(c) shows that there is a comparably intense output together with coupling to the microcavity. Hence, this structure too shows poor performance at resonance. Therefore, for the hexagonal lattice structure of AlAs the best optical switching can be obtained for rods of radius 0.2050 a with a resonance wavelength of $2.393 \mu \mathrm{m}$ when the microcavity is at a distance 2 a from the waveguide.

\section{Square and Hexagonal PC Structure Switches of AIAs}

Both proposed square and hexagonal crystal structures show optimum results, when the microcavity is $2 \mathrm{a}$ away from the waveguide and these are presented in Fig. 7. Square structure exhibits a very sharp resonance drop compared to the hexagonal structure. Further, average normalized power through the waveguide is high in the square lattice for other wavelengths other than the point of resonance. The wavelength range that the square lattice can be used as a switch is larger compared to that of the hexagonal lattice. Hence, the square structure is highly preferred than the hexagonal lattice structure for AlAs switch. 


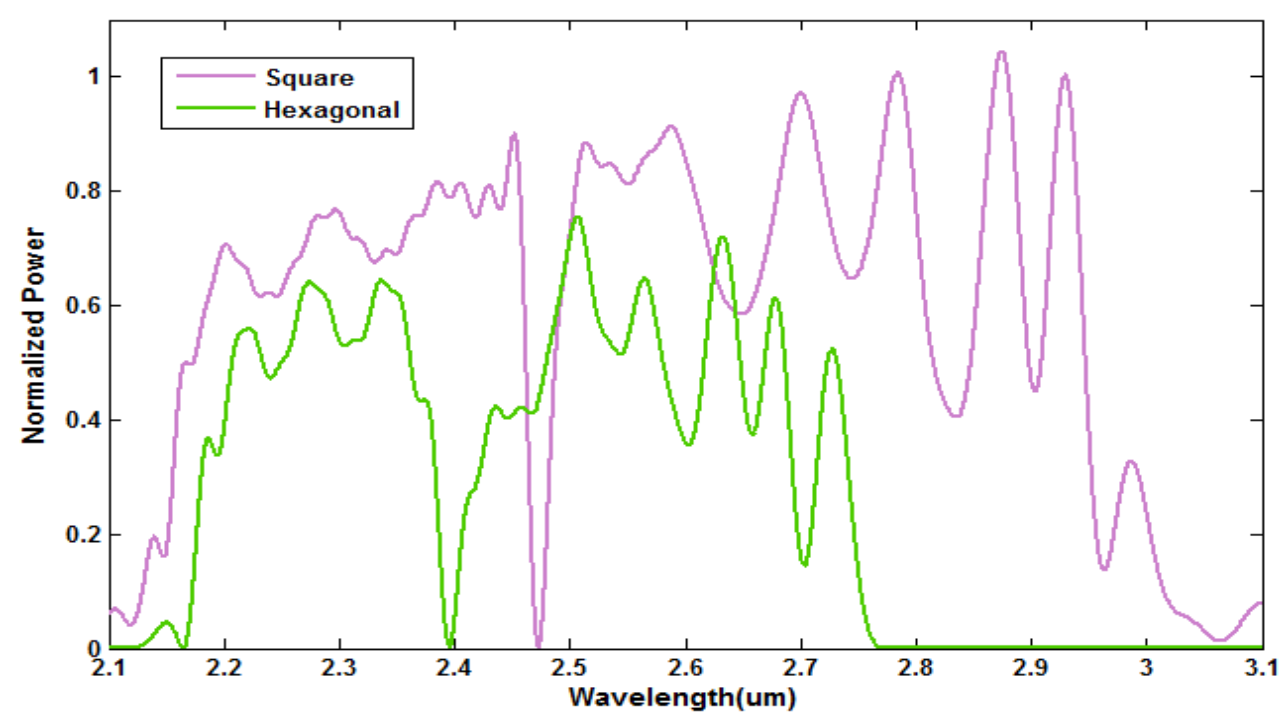

Figure 7. The normalized transmission spectra for the square and hexagonal PC lattice switch structures for microcavity distance 2 a from the middle of the waveguide.

\section{Nonlinear Situation for AIAs}

When Kerr's nonlinearity is present in the medium, as the intensity of the pump signal is increased, the refractive index of the dielectric rods increases. Hence, the optimal situation for the square AlAs PC structure with the microcavity at $2 \mathrm{a}$ from the middle of the waveguide was obtained for nonlinear case by plotting the normalized transmittance curves for the hypothetical refractive index change range of $0.1-0.6$. These with linear case (in red) are shown in Fig. 8. The resonance wavelength increases as the intensity of the pump pulse which is proportional to the refractive index change increases. For the refractive index changes of $0.3-0.5$ resonance is in one range whereas for the refractive index changes in the range $0.0-0.2$ shows resonance in another range. With the increase of nonlinearity the transmission at the resonance slowly increases. The best nonlinear case should contain its resonance wavelength away from the linear resonance as well as it should show a good transmission at the point of resonance wavelength as that of the linear case. Hence the best nonlinear PC structure that may act as a switch will correspond to the refractive index change in the range $0.3-0.5$. The resonance frequencies and the transmittance at the linear resonance frequency of the nonlinear instances are tabulated in table 3.

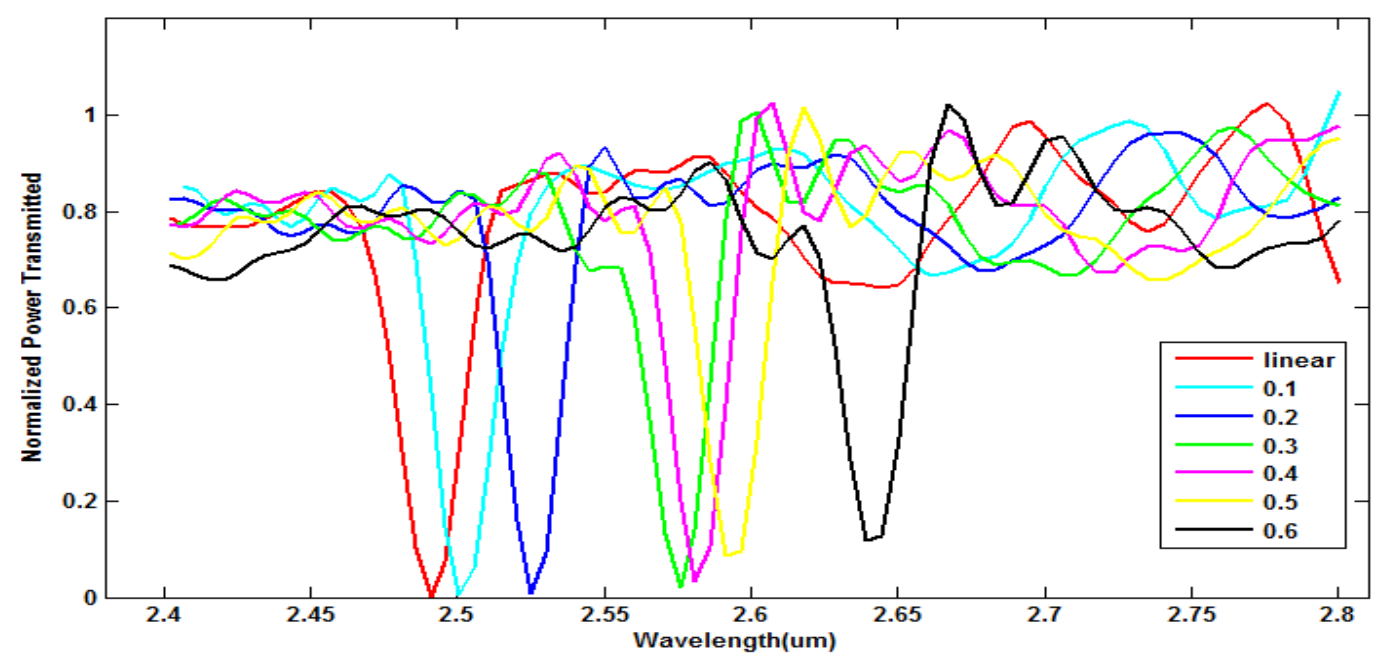

Figure 8. Normalized power transmission spectrum through the waveguide with the increase of refractive index change. The red line shows the linear case whereas other lines depict the behaviour in nonlinear situation. 
Although for the 0.2 change in the refractive index, delivers a high output, Fig. 8 shows that the resonance drop off overlaps with the linear situation. Hence, a small difference in the refractive index change about the 0.2 may give adverse results. Therefore 0.3 to 0.5 range which shows a stable power output with their resonance wavelengths away from the linear resonance wavelength can be considered as the optimal range.

\section{Performance of the Proposed Structure as an Optical Limiter}

Table 3. Nonlinear AlAs optimal square lattice PC structure with microcavity 2a away from the waveguide with refractive indices, corresponding resonance wavelengths and power transmitted.

\begin{tabular}{|c|c|c|c|}
\hline $\begin{array}{c}\text { Refractive index } \\
\text { change }\end{array}$ & $\begin{array}{c}\text { Resonance wavelength } \\
(\mu \mathrm{m})\end{array}$ & $\begin{array}{c}\text { Normalized transmission at } \\
\text { its own resonance }\end{array}$ & $\begin{array}{c}\text { Normalized transmission at } \\
2.4708 \mu \mathrm{m} \text { (resonance of linear) }\end{array}$ \\
\hline 0.1 & 2.5006 & $5.169 \times 10^{-3}$ & 0.4264 \\
\hline 0.2 & 2.5251 & $7.3638 \times 10^{-3}$ & 0.8218 \\
\hline 0.3 & 2.5758 & $2.0546 \times 10^{-2}$ & 0.7715 \\
\hline 0.4 & 2.5809 & $3.1754 \times 10^{-2}$ & 0.7337 \\
\hline 0.5 & 2.5914 & $8.4818 \times 10^{-2}$ & 0.7533 \\
\hline 0.6 & 2.6393 & 0.1166 & 0.8039 \\
\hline
\end{tabular}

From the best switching parameters obtained for AlAs square PC structure, the resonance wavelength at the linear instance was evaluated for a range of nonlinear situations. The intensity of the incoming pulse with a wavelength coinciding with the resonance frequency of the linear case was increased gradually and the normalized transmittance at the end of the waveguide was measured. The intensity of the incoming signal is represented by the corresponding refractive index as defined by Kerr's nonlinearity. These are shown in Fig. 9. The output power of AlAs is gradually increased with the increase input power denoted in terms of refractive index change and the transmittance stabilizes at the refractive index change of 0.07 .

The extinction ratio was obtained as 2.89 by considering the normalized transition at resonance and the stabilization transmittance in nonlinear instance in log scale for the proposed limiter.

Extinction Ratio for a range of refractive indices were obtained for comparison purpose as given in table below.

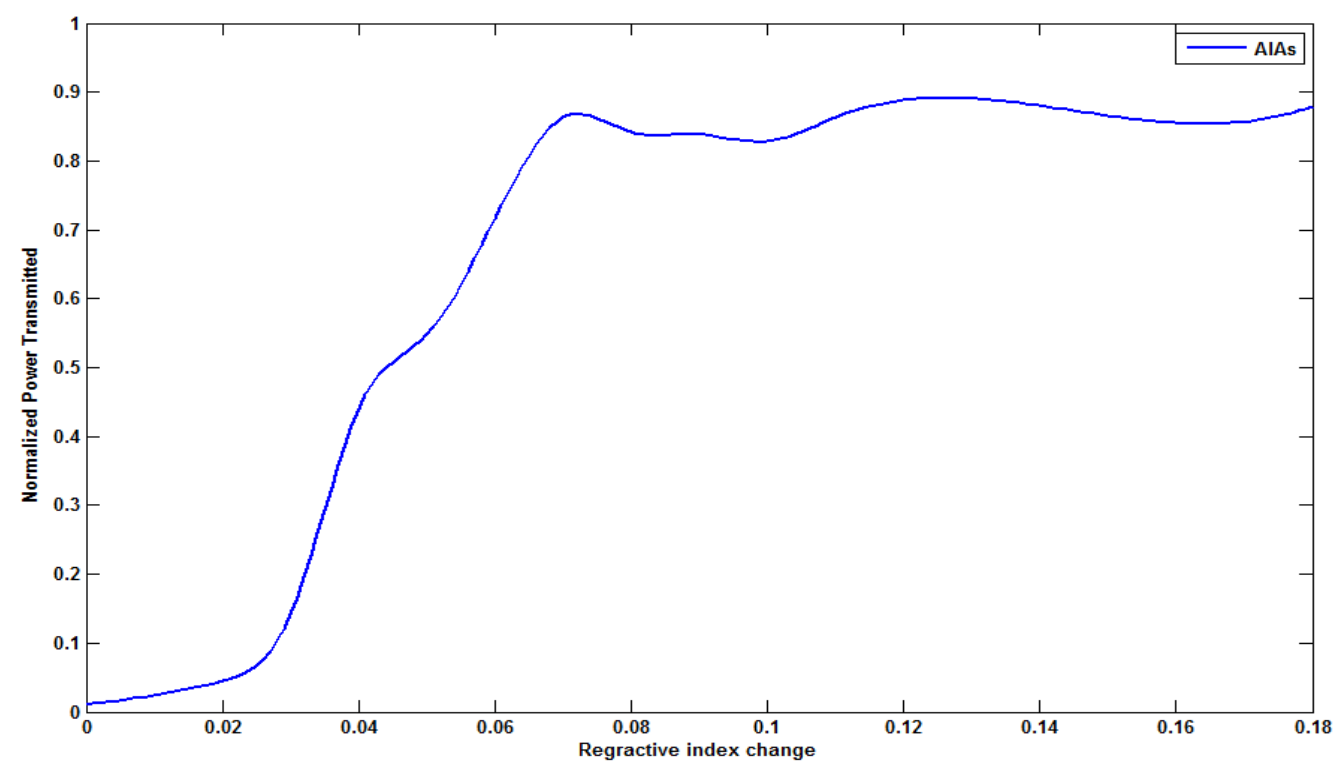

Figure 9. Behaviour of the normalized transmittance through the waveguide at linear resonance frequencies of the optimal structure of AlAs with the increase in input power (denoted in terms of refractive index changes). 
Table 4. Extinction ratio for refractive indexes in log scale for square PC lattice structure with microcavity at a distance ' $2 a$ ' from the center of the waveguide.

\begin{tabular}{|c|c|}
\hline Refractive Index & Extinction Ratio \\
\hline 2.50 & 2.34 \\
\hline 2.60 & 2.19 \\
\hline 2.70 & 2.81 \\
\hline 2.80 & 2.89 \\
\hline 2.90 & 1.81 \\
\hline 3.00 & 1.92 \\
\hline 3.10 & 1.63 \\
\hline 3.20 & 1.08 \\
\hline 3.30 & 1.43 \\
\hline 3.40 & 0.93 \\
\hline 3.50 & 0.75 \\
\hline
\end{tabular}

Therefore, AlAs is suitable for the implementation of the proposed limiter structure.

\section{Tuning the Optical Switch and the Limiter}

It is essential to tune the optical switch and the limiter to the desired frequency range. The range of wavelengths dealt with, in the simulations carried so far are in the range of $2.0 \mu \mathrm{m}$ to 4.00 $\mu \mathrm{m}$. However, depending on the necessity it is essential to have the flexibility to tune the switch and the limiter to any required wavelength range. For this, the band gap of the photonic crystal needs to be shifted to the desired wavelength range. The variation of bandgaps with the lattice constants of square AlAs lattice is shown in Fig. 10. By changing the lattice constant, the frequency band of operation can be altered. Hence, photonic crystals possess the ability to be tuned to the required operational frequency. For the AlAs square lattice with the optimal radius of AlAs rods $0.1875 \mathrm{a}$, the bandgap obtained with lattice constant of $1 \mu \mathrm{m}$ is $0.345 \leq 1 / \lambda \leq 0.457$ and the mid gap lies at $0.401 \lambda^{-1}$. If the crystal needs to be tuned to work at the most commonly used telecommunication wavelength $1.55 \mu \mathrm{m}$, it should be made to operate in the middle of the gap. For this,

$$
\frac{\omega \mathrm{a}}{2 \pi c}=\frac{\mathrm{a}}{\lambda}=\frac{\mathrm{a}}{1.55 \mu \mathrm{m}}=0.401 \Rightarrow \mathrm{a}=0.6015 \mu \mathrm{m}
$$

The corresponding bandgap for the above lattice constant lies in the range $0.5943 \leq 1 / \lambda \leq 0.7722$ and the mid gap is at $0.68325 \lambda^{-1}$. The wavelength range corresponding to the band gap is $1.2950 \mu \mathrm{m}-1.6827 \mu \mathrm{m}$ which includes the telecommunication wavelength of $1.55 \mu \mathrm{m}$. Therefore, AlAs photonic crystals can be fine-tuned to the range of frequencies need to be operated. 


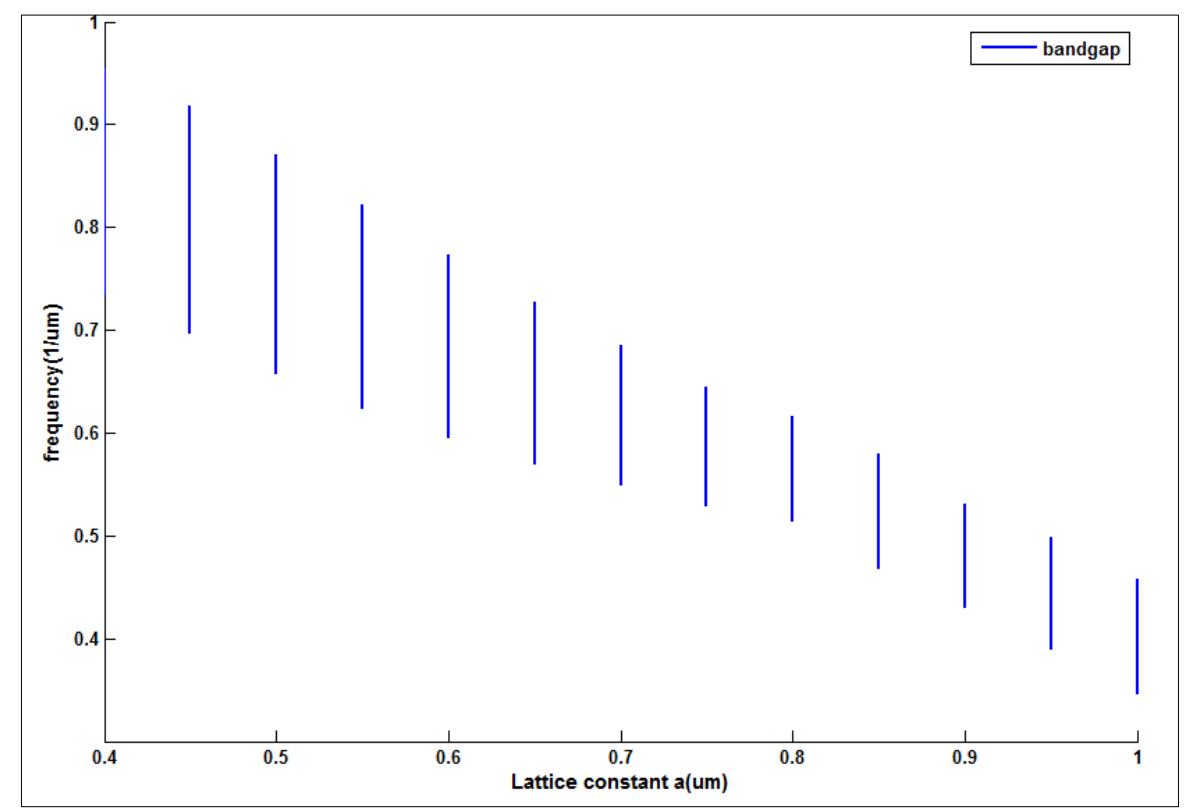

Figure 10. Variation of band gap with the lattice constant for AlAs square lattice with refractive index 2.892 .

\section{Conclusions}

For the proposed structure, when the input signal frequency is equal to the resonant frequency of the microcavity, the output through the waveguide becomes negligibly small. In the nonlinear case due to Kerr's nonlinearity the resonant frequency of the cavity red shifts, as a result the signal transmits through the waveguide producing a considerable output. With the increase of microcavity distance from the waveguide the resonant frequency of the microcavity decreases. Square lattice structure is more preferable over the hexagonal lattice for the implementation of the switch and the limiter structures with the microcavity situated $2 \mathrm{a}$ away from the centre of the waveguide. The best nonlinear situation for the proposed AlAs square lattice PC structure to be active as a switch will be corresponding to the refractive index change 0.3 to 0.5 with cylindrical rod radius of $0.1875 \mathrm{a}$. The extinction ratio of the limiter was obtained as 2.09. The proposed switch can be fine-tuned to work under the intended frequency by altering the lattice constant of the structure. The lattice constant for the proposed structure to work at the telecommunication wavelength of $1.55 \mu \mathrm{m}$ is $0.6015 \mu \mathrm{m}$.

\section{References}

[1] J.D. Joannopoulos, M. Soljacic, Enhancement of nonlinear effects using photonic crystals, Nature Materials. 3(4) (2004) 211-219.

[2] J.D. Joannopoulos et al., Photonic crystals molding the flow of light, 2nd ed., New Jersey: Princeton University press, 2008.

[3] J.C. Attila Mekis et al., High Transmission through Sharp Bends in Photonic Crystal Waveguides, Physical Review Letters. 77(18) (1996) 3787-3790.

[4] A. Mekis, S. Fan, J.D. Joannopoulos, Bound states in photonic crystal waveguides and waveguide bends, Physical Review B. 58(8) (1998) 4809-4817.

[5] P.H.F. Ramos-Mendieta, Tunable photonic crystals with semiconducting constituents, Physical Review Letters. 85(9) (2000) 1875-1878.

[6] A. Figotin, Y.A. Godin, I. Vitebsky, Two-dimensional tunable photonic crystals, Physical Review B. 57(5) (1998) 2841-2848.

[7] P. A. Franken et al., Generation of optical harmonics, Physical Review Letters. 7(4) (1961) 118-120. 
[8] P. Tran, Optical switching with a nonlinear photonic crystal: a numerical study, Optics Letters. 21(15) (1996) 1138-1140.

[9] M. Danaie, H. Kaatuzian, Employing optical nonlinearity in photonic crystals: a step towards all-optical logic gates, in: Photonic Crystals-Innovative Systems, Lasers and Waveguides, InTech, 2012.

[10] M. Scalora et al., Optical limiting and switching of ultrashort pulses in nonlinear photonic band gap materials, Phys. Rev. Lett. 73(10) (1994) 1368-1371.

[11] S. Scholz, O. Hess, R. Rühle, Dynamic cross-waveguide optical switching with a nonlinear photonic band-gap structure, Optics Express. 3(1) (1998) 28-34.

[12] A. T. Rahmati, N. Nozhat, N. Granpayeh, Photonic crystal switches based on nonlinear directional couplers, in: 18th Iranian Conference on Electrical Engineering (ICEE), Isfahan, 2010 .

[13] M.F. Yanik et al., All-optical transistor action with bistable switching in a photonic crystal cross-waveguide geometry, Optics Letters. 28(24) (2003) 2506-2508.

[14] N. Nozhat, A.T. Rahmati, N. Granpayeh, An all optical switch based on nonlinear photonic crystal microcavities, in: Progress in Electromagnetics Research Symposium (PIERS) Proceedings, Moscow, Russia, August 18-21, 2009.

[15] A. Locatelli et al., All optical switching in ultrashort photonic crystal couplers, Optics Communications. 237 (2004) 97-102.

[16] A.T. Rahmati, N. Granpayeh, Simulation of an ultrashort 2D photonic crystal switch based on nonlinear directional coupler, in: Progress in Electromagnetics Research Symposium (PIERS) Proceedings, Moscow, Russia, August 18-21, 2009.

[17] I.V. Guryev et al., Optical power limiter on the basis of 2D photonic crystal, in: Advanced Optoelectronics and Lasers, CAOL 2008, 4th International Conference, September 29 October 4, Alushta, Crimea, Ukraine, 2008.

[18] M. Danaie, H. Kaatuzian, H. S. Kojori, Design of a photonic crystal optical limiter using nonlinear Kerr effect, in: Proc. SPIE 8205, 2011 International Conference on Photonics, 3DImaging, and Visualization, Guangzhou, China, 2011.

[19] M. Danaie, H. Kaatuzian, Design and simulation of an all-optical photonic crystal AND gate using nonlinear Kerr effect, Optical and Quantum Electronics. 44(1) (2012) 27-34.

[20] A. Naqavi et al., Optical bistable switching with Kerr nonlinear materials exhibiting a finite response time in two-dimensional photonic crystals, in: Proc. SPIE 7713, Photonic Crystal Materials and Devices IX, 77131T, Belgium, 2010.

[21] K. Yee, Numerical solution of initial boundary value problems involving Maxwell's equations in isotropic media, IEEE Transactions on Antennas and Propagation. 14(3) (1966) 302-307.

[22] S. Gedney, Introduction to the finite-difference time-domain (FDTD) method for electromagnetics, Arizona: Morgan \& Claypool Publishers, 2011.

[23] R.W. Boyd, Nonlinear optics, Third Edition, New York: Academic Press, 2007.

[24] M.H. Holmes, Multiple scales, in: Introduction to Perturbation Methods, London, Springer, 2013, p. 145.

[25] J.P. Berenger, A perfectly matched layer for the absorption of electromagnetic waves, Journal of Computational Physics. 114(2) (1994) 185-200. 
[26] J.C. Veihl, R. Mittra, Efficient implementation of Berenger's perfectly matched layer (PML) for finite-difference time-domain mesh truncation, IEEE Microwave and Guided Wave Letters. 6(2) (1996) 94-96.

[27] J.P. Berenger, Perfectly matched layer for the FDTD solution of wave-structure interaction problems, IEEE Transactions on Antennas and Propagation. 44(1) (1996) 110-117.

[28] Z.S. Sacks et al., A perfectly matched anisotropic absorber for use as an absorbing boundary condition, IEEE Transactions on Antenas and Propergation. 43(12) (1995) 1460-1463.

[29] I. Maksymov, Modelling of photonic components based on nonlinear photonic crystals, Thesis, Rovira i Virgili University, 2010.

[30] R.E. Fern, A. Onton, Refractive index of AlAs, J. Appl. Phys. 42(9) (1971) 3499-3500. 